Supplement of Geochronology, 3, 171-180, 2021

https://doi.org/10.5194/gchron-3-171-2021-supplement

(c) Author(s) 2021. CC BY 4.0 License.

(c) (i)
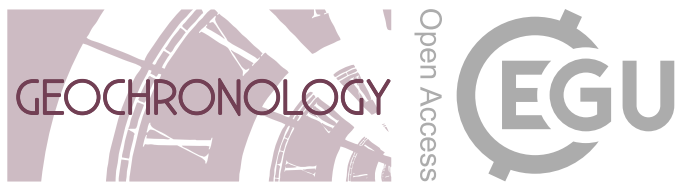

Supplement of

\title{
Short communication: Driftwood provides reliable chronological markers in Arctic coastal deposits
}

Lasse Sander et al.

Correspondence to: Lasse Sander (lasse.sander@awi.de)

The copyright of individual parts of the supplement might differ from the article licence. 


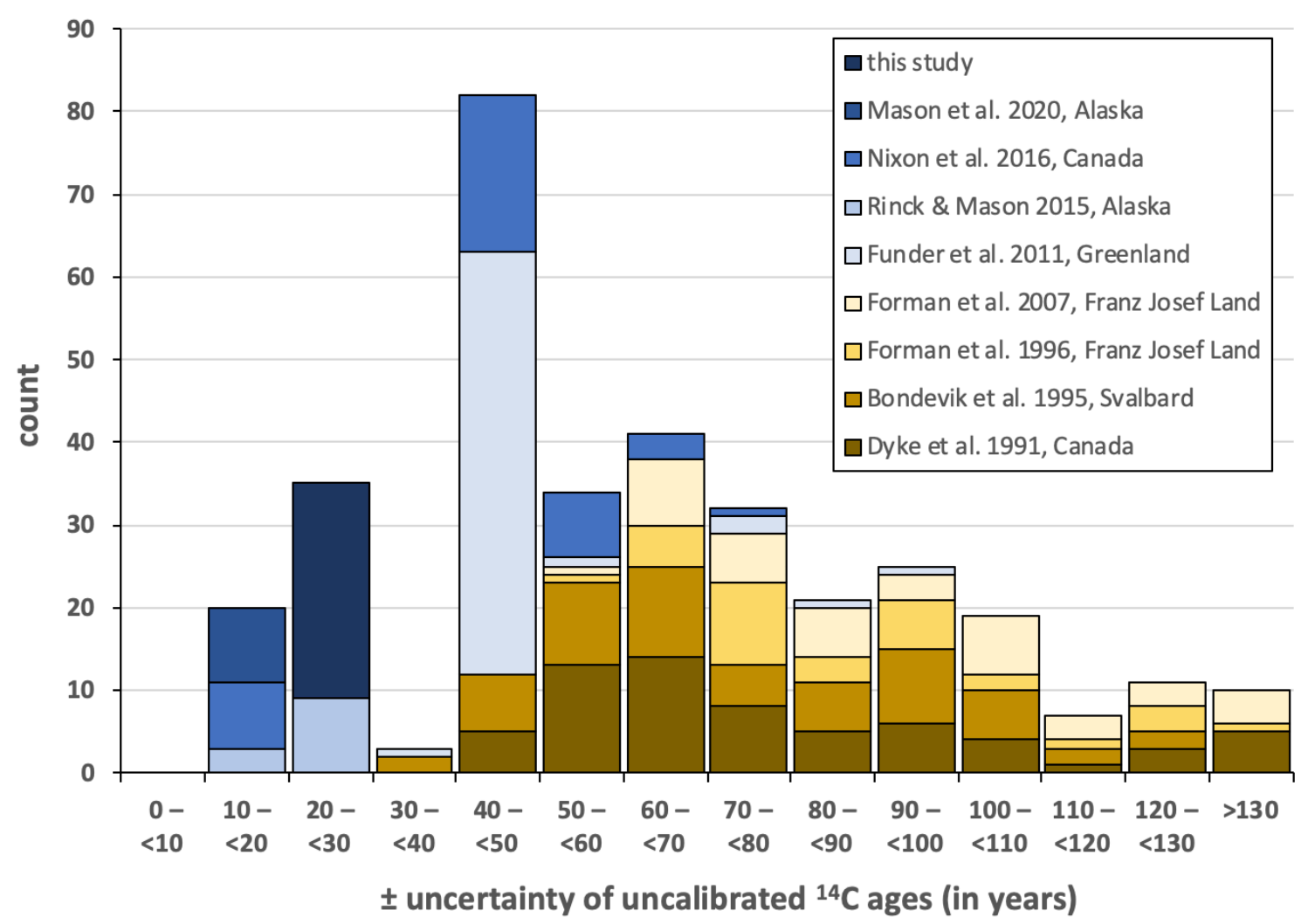

Figure S1: Overview of changes in the range of uncertainty in the ${ }^{14} \mathrm{C}$ age of driftwood as

5 reported in selected studies in Arctic coastal contexts. The data are displayed as uncalibrated samples and the figure illustrates that technological advancements in the ${ }^{14} \mathrm{C}$ method continually improved the quality of the age determination. Current uncertainties are on the order of \pm 15 years (e.g. Wacker et al. 2010) and thus in most cases shorter than uncertainties stemming from an unknown sample position within the tree-ring chronology or an unaccounted weathering loss 10 (cf. Fig. S4). 


\section{Plot ("Bys_Tasa")}

\{P_Sequence("Bys_Tasa",0.5)

15 Boundary ("innermost_ridge") $\{z=0 ;\}$;

R_Date ("BY-1" , 5425, 30) $\{\mathrm{z}=0$; $\}$;

R_Date ( "BY-2" , 5441,30) $\{z=0$; ;

R_Date ( $B Y-6 ", 5063,29)\{z=130$; $\}$;

R_Date ( $B Y-5$ " ,5118, 29) $\{z=140$; ;

20 R_Date( "BY-7" "4697, 29) $\{z=240 ;\}$;

R_Date ( $B Y-3 ", 4387,29)\{z=400$; $\}$;

R_Date ( $B Y-4 ", 4380,29)\{z=400 ;\}$;

R_Date ( "K3" , 4038,49) $\{z=510 ;\}$;

R_Date ( "K4 ", 3889, 49) $\{z=590 ;\}$;

25 R_Date( "BY-8", 3544, 28) $\{z=700 ;\}$;

R_Date ( "K2" , 3500, 48) $\{z=700$; ;

R_Date ( "K1" , 3206, 48) $\{z=880 ;\}$;

Boundary (“disconformity) $\{z=1100 ;\} ;\}$;

Figure S2: OxCal code for the age model of the Bys Tasa (BY) beach-ridge system 30 


\section{Plot ("Makhchar")}

\{P_Sequence ("Makhchar",0.5)

\{Boundary ("innermost_ridge") $\{z=0 ;\}$;

$$
\text { R_Date ("MA-40",5127,29) }\{z=0 ;\} \text {; }
$$

R_Date( $" M A-41 ", 5294,29)\{z=0 ;\}$;

R_Date ("MA-42",5153,29) $\{z=0 ;\}$;

R_Date ("MA-32",4394,28) $\{z=280 ;\}$;

R_Date ("MA-33",4322,29) $\{z=280 ;\}$;

R_Date ("MA-30",4382,29) $\{z=315 ;\}$;

R_Date ("MA-31",4344,29) $\{z=315 ;\}$;

R_Date( "MA-27",4149,28) $\{z=410$; ;

R_Date ("MA-28", 4169,28) $\{z=410$; ;

R_Date( "MA-29",4175, 28) $\{z=410$; ;

Boundary ( "marked_ridge 1 ") $\{z=810 ;\}$;

45

R_Date ( "MA-39" ,2121,28) $\{z=860$; ;

Boundary ( "marked_ridge 2 ") $\{z=890$; $\}$;

R_Date ( "MA-4" , 1133,27) $\{z=915$; $\}$;

R_Date ("MA-3", 944,27$)\{z=940 ;\}$;

R_Date ("MA-1" , 770,27) $\{z=965 ;\}$;

50

R_Date ("MA-26",568,27) $\{z=970 ;\}$;

Boundary ("stormberm") $\{\mathrm{z}=980 ;\} ;\} ;\}$;

Figure S3: OxCal code for the age model of the Makhchar (MA) beach-ridge system 


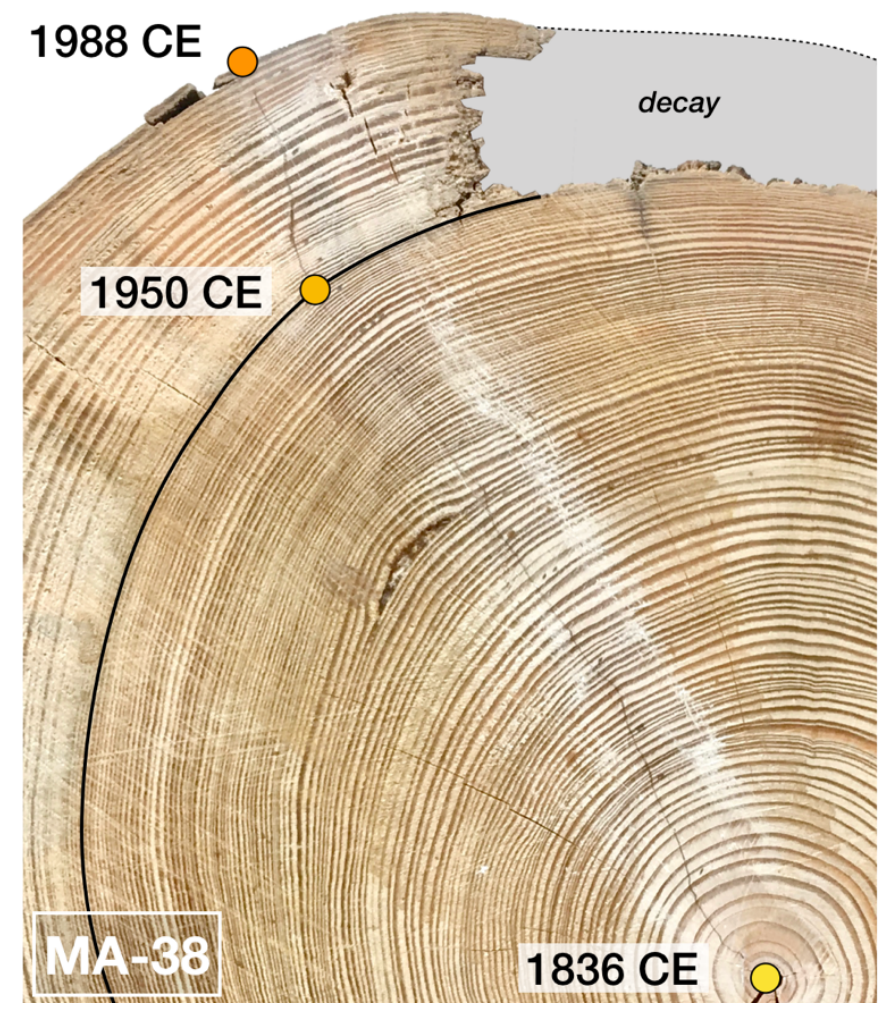

55 Figure S4: The decay of tree rings can produce significant errors in the age determination. This figure is to illustrate, how an unknown position of a ${ }^{14} \mathrm{C}$ sample in relation to the outermost treering might affect the quality of the established chronology (and hence the paleoenvironmental interpretation). Complete radius: $9.3 \mathrm{~cm}$ 

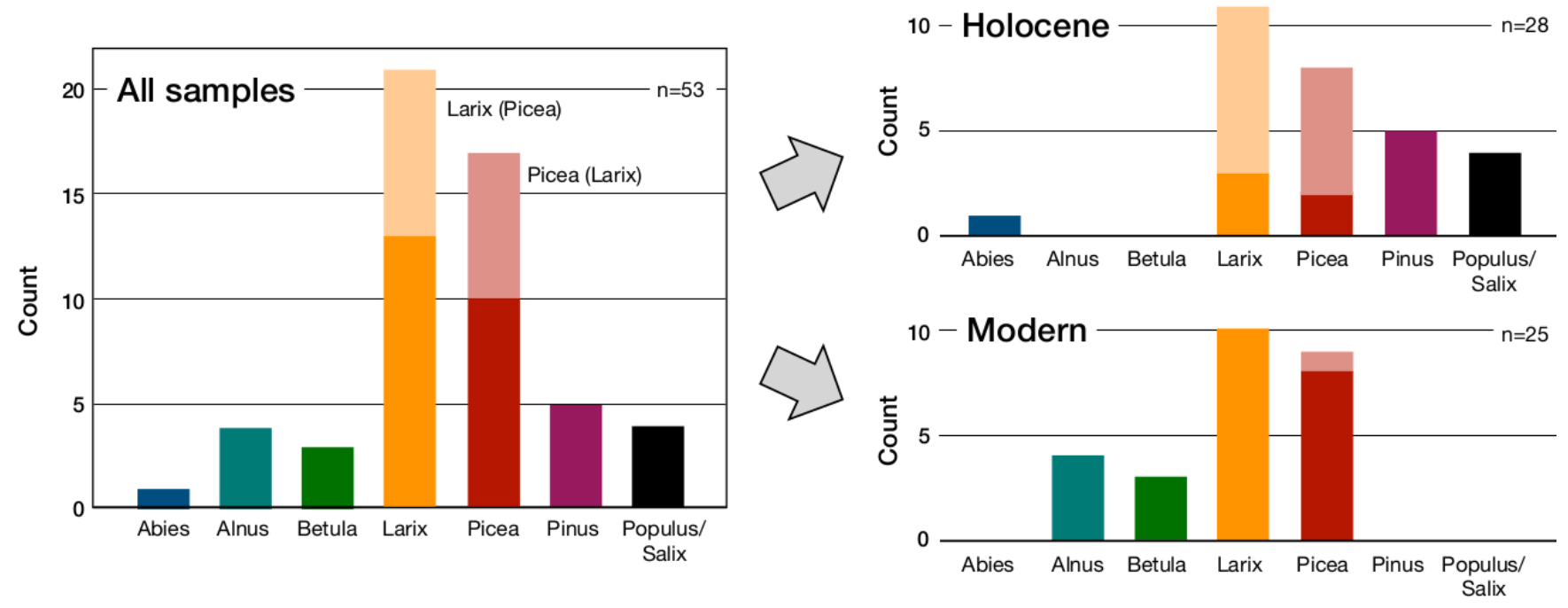

Figure S5: Composition of the sample population based on the genus of the driftwood samples. 
Table S1: Results of the Bayesian age model for Bys Tasa

\begin{tabular}{|c|c|c|c|c|c|}
\hline ID & Set & $\begin{array}{r}\text { Unmodelled age* } \\
\text { (Probability, in \%**) }\end{array}$ & $\begin{array}{r}\text { Modelled age } \\
\text { (Probability, in \%**) }\end{array}$ & $\begin{array}{r}\text { Age span, modelled } \\
\text { (unmodelled) }\end{array}$ & Agreement \\
\hline Boundary & & $\mathrm{N} / \mathrm{A}$ & $4340-4250$ BCE (95.4) & $90(\mathrm{~N} / \mathrm{A})$ & $\mathrm{N} / \mathrm{A}$ \\
\hline BY-1 & 1 & $4350-4230$ BCE (95.4) & $4340-4260$ BCE (95.4) & $80(120)$ & 112.1 \\
\hline BY-6 & 2 & $3960-3790$ BCE (95.4) & $3920-3820$ BCE (95.4) & $100(170)$ & 104.8 \\
\hline BY -5 & 2 & $\begin{array}{l}3980-3910 \text { BCE (46.3) } \\
3880-3800 \text { BCE }(49.1)\end{array}$ & $3880-3800$ BCE (95.4) & $80(180)$ & 85.0 \\
\hline BY -3 & 3 & $3100-2920$ BCE (95.4) & $3020-2920$ BCE (95.4) & $100(180)$ & 114.1 \\
\hline BY-4 & 3 & $3090-2910$ BCE (95.4) & $3020-2920$ BCE (95.4) & $100(180)$ & 114.6 \\
\hline K3 & - & $2700-2460$ BCE (86.3) & $2660-2500$ BCE (95.4) & $160(240)$ & 111.6 \\
\hline K4 & - & $2480-2200$ BCE (95.4) & $2390-2220$ BCE (95.4) & $170(2280)$ & 91.5 \\
\hline K1 & - & $1620-1400(95.4)$ & $\begin{array}{l}1510-1370(70.4) \\
1360-1290(25.0)\end{array}$ & $220(220)$ & 68.2 \\
\hline Boundary & & N/A & $880-460$ BCE (95.4) & $420(\mathrm{~N} / \mathrm{A})$ & N/A \\
\hline
\end{tabular}

$*$ rounded to tenth

$70 * *$ probabilities $<10 \%$ are not displayed and not considered in the calculation of the age span 
Table S2: Results of the Bayesian age model for Makhchar

\begin{tabular}{|c|c|c|c|c|c|}
\hline ID & Set & $\begin{array}{r}\text { Unmodelled age } \\
\text { (Probability, in \%) }\end{array}$ & $\begin{array}{r}\text { Modelled age } \\
\text { (Probability, in \%) }\end{array}$ & $\begin{array}{r}\text { Age span, modelled } \\
\text { (unmodelled) }\end{array}$ & Agreement \\
\hline Boundary & & N/A & $4040-3960(95.4)$ & $80(\mathrm{~N} / \mathrm{A})$ & $\mathrm{N} / \mathrm{A}$ \\
\hline MA-40 & 5 & $\begin{array}{l}3990-3910 \text { ВCE }(55.1) \\
3880-3800 \text { ВCE }(40.3)\end{array}$ & $4040-3960$ BCE (95.4) & $80(190)$ & 28.4 \\
\hline MA-41 & 5 & $4240-4040$ BCE (95.0) & $4040-3960$ BCE (95.4) & $80(200)$ & 3.7 \\
\hline MA-42 & 5 & $4050-3930$ BCE (86.1) & $4040-3960$ BCE (95.4) & $80(120)$ & 79.0 \\
\hline MA-32 & 6 & $3100-2920$ BCE (95.4) & $\begin{array}{l}3100-3050 \text { BCE (61.7) } \\
3030-2990 \text { BCE }(33.7)\end{array}$ & $110(210)$ & 110.5 \\
\hline MA-33 & 6 & $3020-2890$ BCE (95.4) & $\begin{array}{l}3090-3050 \text { BCE (61.9) } \\
3030-2990 \text { BCE (33.5) }\end{array}$ & $100(130)$ & 17.1 \\
\hline MA-30 & 7 & $3090-2910$ BCE (95.4) & $3010-2910$ BCE (95.4) & $100(180)$ & 96.1 \\
\hline MA-31 & 7 & $3030-2890$ BCE (94.7) & $3010-2910$ BCE (95.4) & $100(140)$ & 96.0 \\
\hline MA-27 & 8 & $2880-2630$ BCE (95.4) & $2780-2660$ BCE (93.2) & $120(250)$ & 112.8 \\
\hline MA-28 & 8 & $\begin{array}{l}2890-2830 \text { BCE (19.8) } \\
2820-2660 \text { BCE }(73.3)\end{array}$ & $2780-2660$ BCE (93.1) & $120(230)$ & 100.9 \\
\hline MA-29 & 8 & $\begin{array}{l}2890-2830 \text { BCE }(20.7) \\
2820-2660 \text { BCE }(74.7)\end{array}$ & $2780-2660$ BCE (93.1) & $120(230)$ & 91.9 \\
\hline Boundary & & N/A & $1720-1180$ BCE (95.4) & $540(\mathrm{~N} / \mathrm{A})$ & $\mathrm{N} / \mathrm{A}$ \\
\hline MA-39 & - & $210-50$ BCE $(92.1)$ & $210-50$ BCE (95.4) & $160(160)$ & 101.7 \\
\hline Boundary & & N/A & $510-880$ CE (95.4) & $370(\mathrm{~N} / \mathrm{A})$ & $\mathrm{N} / \mathrm{A}$ \\
\hline MA-4 & 9 & $860-990$ CE (87.4) & $810-990$ CE (93.7) & $180(130)$ & 101.1 \\
\hline MA-3 & 9 & $1020-1160$ CE (95.4) & $1030-1150$ CE (95.4) & $120(140)$ & 102.5 \\
\hline MA-1 & 9 & $1210-1280 \mathrm{CE}(95.4)$ & $1230-1290 \mathrm{CE}(95.4)$ & $60(70)$ & 108.7 \\
\hline MA-26 & 9 & $\begin{array}{l}1300-1370 \mathrm{CE}(56.2) \\
1380-1430 \mathrm{CE}(39.2) \\
\end{array}$ & $1290-1360 \mathrm{CE}(95.4)$ & $70(130)$ & 88.6 \\
\hline Boundary & & $\mathrm{N} / \mathrm{A}$ & $1320-1500 \mathrm{CE}(95.4)$ & $180(\mathrm{~N} / \mathrm{A})$ & $\mathrm{N} / \mathrm{A}$ \\
\hline
\end{tabular}

* rounded to tenth

** probabilities $<10 \%$ are not displayed and not considered in the calculation of the age span 